due to peer pressure, $35.2 \%$ consumed tobacco as they felt better, and $5 \%$ consumed tobacco to ease abdominal complaints and dental problem. The reasons for non use of tobacco among girls were fear of cancer (59\%) and poor oral health (37.9\%).

Conclusions The current consumption of any tobacco products among rural adolescents was found very high with multiple protobacco influences. It requires policy level intervention to tackle the problem.

\section{P1-134 DELAYS IN ACCESSING HIV/AIDS HEALTH SERVICES PERSIST IN SALVADOR, BRAZIL}

doi:10.1136/jech.2011.142976d.27

${ }^{1}$ D Dourado, ${ }^{1,2} \mathrm{C}$ Lima, ${ }^{1,3} \mathrm{~S}$ MacCarthy. ${ }^{1}$ Public Health Institute, Federal University of Bahia, Salvador, Bahia, Brazil; ${ }^{2}$ CETAD, Bahia State Department of Health, Salvador, Bahia, Brazil; ${ }^{3}$ Harvard School of Public Health, Boston, Massachusetts, USA

Introduction Even though Brazil was the first country to provide universal and free access to ARVs, substantial regional differences exist in the timing that services and care are accessed. Studies among people living with HIV have documented delays in accessing services, with the largest proportion of delayed access reported in the Northeastern Region. Further investigation is needed to understand the factors contributing to this delay despite the availability of HIV/ AIDS services.

Methods This is a retrospective cohort of patients $(n=1440)$ from the only state reference center located in Salvador, the capital city in the Northeastern state of Bahia. The data were abstracted from the electronic medical records of all new patients between 2007 and 2009, and is supplemented with information from two national databases. The dependent variable, delayed access, is defined as patients accessing services with a CD4 count $<350$ cells $/ \mathrm{mm}^{3}$. The independent variables are based on a comprehensive review of the literature. Frequencies and cross tables informed the final logistic regression model.

Results Nearly $35 \%$ of patients were delayed in accessing HIV/AIDS services. The final logistic regression model $(p$ value $=0.05$ ) showed that individuals who both drank and smoked were 3.4 times more likely to delay in accessing services ( $p$ value $=0.036$ ). Additionally, individuals with a family income of $<1$ minimum wage were 1.9 times more likely to delay in accessing services ( $p$ value $=0.07$ ).

Conclusions Considering this documented delay, it is clear additional efforts are necessary to ensure HIV/AIDS services in Brazil are truly free and universal.

\section{P1-135 ADULT PSYCHOSOCIAL OUTCOMES OF CHILDHOOD PUBLIC CARE: A LIFE COURSE PERSPECTIVE USING THE BCS70 COHORT STUDY}

doi:10.1136/jech.2011.142976d.28

A Dregan, ${ }^{*}$ M Gulliford, D Armstrong. King's College, London, UK

Objectives The extent to which differences in childhood experiences of public care are related to adult psychosocial outcomes is unknown. This study aimed to estimate associations between childhood experiences of the public care system with emotional and behavioural traits at age 30 years.

Methods Participants included 10895 respondents at the age 30 survey of the 1970 British Cohort Study (BCS70) who were not adopted and whose care history was known. Two estimation models were employed to determine whether public care and placement patterns were associated with adult psychosocial outcomes. Analyses were adjusted for individual, parental and family characteristics in childhood.

Results Cohort members with a public care experience reported lower childhood family socio-economic compared to those in the no public care group. After adjusting for confounding, exposure to both foster and residential care, longer placements and multiple placements were associated with more extensive adult emotional and behavioural difficulties. Specifically, residential care was associated with adult criminal convictions (OR $3.09,95 \%$ CI 2.10 to 4.55 ) and adult depression $(1.81,1.23$ to 2.68$)$ compared to no public care placement. Multiple placements were associated with low self-efficacy in adulthood (3.57, 2.29 to 5.56). Admission to care after the age of 10 was associated adult criminal convictions (6.03, 3.34 to 10.90) and smoking (3.32, 1.97 to 5.58).

Conclusion Children who experience public care have impaired wellbeing as adults. Older age at admission, multiple care placements and residential care are associated with worse outcomes.

\section{P1-136 SECOND AND THIRD TRIMESTERS PREGNANCY WEIGHT GAIN: ASSOCIATION WITH MATERNAL AND FETAL OUTCOMES}

doi:10.1136/jech.2011.142976d.29

${ }^{1} \mathrm{M}$ Drehmer, ${ }^{* 1} \mathrm{~B}$ Duncan, ${ }^{2} \mathrm{G}$ Kac, ${ }^{1} \mathrm{M}$ I Schmidt. ${ }^{1}$ Universidade Federal do Rio Grande do Sul. Programa de Pós-Graduação em Epidemiologia., Porto Alegre, Rio Grande do Sul, Brazil; ${ }^{2}$ Universidade Federal do Rio de Janeiro. Instituto de Nutrição., Rio de Janeiro, Rio de Janeiro, Brazil

Background The Institute of Medicine (IOM) has recently made new recommendations for total and trimester gestational weight gain. However, deviates from recommended weight gain according to gestational trimester have been little investigated with regard to obstetric outcomes.

Objectives To investigate association between weekly weight gain during second and third trimesters, and maternal and fetal outcomes, taking into account pregestational body mass index and other variables.

Methods Gestational weight gain was evaluated in 3063 pregnant women of the Brazilian Study on Gestational Diabetes (Estudo Brasileiro do Diabetes Gestacional-EBDG) and classified according to the last IOM recommendation. Outcomes were cesarean delivery, preterm birth and small or large for gestational age. Associations between adequate weight gain and outcomes were estimated using Poisson regression. Possible confounders were pregestational body mass index, age, height, race, parity, education, smoking, alcohol consumption, diabetes and hypertensive disorders.

Results In fully adjusted models, second trimester insufficient weight gain was associated with small for gestational age (RR 1.55 95\% CI 1.19 to 2.01); second trimester excessive weight gain with large for gestational age (RR $1.5895 \%$ CI 1.20 to 2.08); third trimester insufficient weight gain with preterm birth (RR $1.5595 \%$ CI 1.08 to 2.22). Third trimester excessive weight gain was associated with preterm birth (RR $1.5495 \%$ CI 1.07 to 2.21) and cesarean delivery (RR 1.17 95\% CI 1.04 to 1.33).

Conclusion Deviates from recommended weight gain during each trimester are associated with adverse pregnancy outcomes. These findings support the IOM recommendations for nutritional care during pregnancy.

\section{P1-137 RACIAL DIFFERENCES IN INFANT MORTALITY: ANALYSIS OF AVOIDABLE CHILD DEATHS IN BELO HORIZONTE, BRAZIL (2000-2007)}

doi:10.1136/jech.2011.142976d.30

${ }^{1}$ E Drumond, ${ }^{*}{ }^{2} \mathrm{C}$ Cunha, ${ }^{2} \mathrm{D}$ Abreu. ${ }^{1}$ Municipal Health Department, Belo Horizonte, Minas Gerais, Brazil;, ${ }^{2}$ Federal University of Minas Gerais, Belo Horizonte, Minas Gerais, Brazil

Infant mortality (IM) is an important health index. Avoidable deaths are those preventable through effective health services. 